\title{
Femtoscopy in heavy ion collisions: Wherefore, whence, and whither? ${ }^{\wedge}$
}

\author{
Mike Lisa \\ Physics Department, Ohio State University, 191 W. Woodruff Ave,, Columbus Ohio 43210, USA
}

\begin{abstract}
I present a brief overview of the wealth of femtoscopic measurements from the past two decades of heavy ion experiments. Essentially every conceivable "knob" at our disposal has been turned; the response of two-particle correlations to these variations has revealed much about the space-momentum substructure of the hot source created in the collisions. I discuss the present status of the femtoscopic program and questions which remain, and point to new efforts which aim to resolve them.
\end{abstract}

The slowly crawling ants will eat our dreams.

Andre Białas, musing on words of Andre Breton as they might apply to femtoscopy.

Go to the ant, thou sluggard; consider her ways, and be wise. - Proverbs vi.6

\section{WHEREFORE}

High energy collisions between electrons, hadrons, or nuclei produce highly nontrivial systems. Especially in the soft (low- $p_{T}$, long spatial scale) sector, the inclusive distributions of the measured multiparticle final states are dominated by phase-space; to first order the momentum spectra and particle yields appear thermal, revealing little of the underlying physics of interest. Detailed information in this sector is obtained only through correlations; inclusive spectra tell much less than half the story.

In particular, multiparticle production is a dynamic process, evolving in space and time. For several decades now, small relative momentum two-particle correlations have been used to probe the space-time structure of systems at the femtometer scale. Measurements and constantly-improving techniques variously called "intensity interferometry," "HBT," "GGLP," "non-identical correlations," etc, are nowadays discussed under the common rubric of femtoscopy [1], as the title of this new workshop series reflects.

While understanding the space-time features of the system is important to both the particle and the heavy ion physicist, in the latter case it is even vital. After all, nontrivial geometrical effects dominate the physics of heavy ion collisions.

From the very broadest perspective, the entire heavy ion program is geared to generate and study a qualitative change in the geometric substructure of the hot system. Strongly-

\footnotetext{
${ }^{1}$ Combined write-up of presentations at XXXV International Symposium on Multiparticle Dynamics, and at the Workshop on Particle Correlations and Femtoscopy, Kroměříž, Czech Republic, August 2005
} 
coupled [2] or not, the quark-gluon plasma (QGP) is a soft QCD system, in which colored degrees of freedom are relevant over large length scales. Of particular interest is the existence and nature of a deconfinement phase transition; a significant and sudden change in the degrees of freedom should be reflected in space-time aspects of the system [3]. Also of generic importance is the (often unasked) question of whether the "system" generated is, indeed, a system. Any discussion of "matter" or "bulk" properties relies on an affirmative answer.

More specifically, geometry defines each stage of the system's evolution. In the initial state, the entrance-channel geometry (impact parameter $\vec{b}$ ) determines the subsequent collective evolution and anisotropic expansion of the system [4]; the resulting "elliptic flow" [5] has been the basis of $\sim 25 \%$ of the publications from the RHIC program. In the intermediate state, also, geometry dominates: quantitative understanding of exciting parton energy loss (or "jet quenching") measurements [6, 7] requires detailed information of the evolving size and anisotropic shape of the system. If coalescence is indeed the mechanism of bulk hadronization [8], space-momentum correlations in the intermediate stage induce clustering effects which must be modeled quantitatively [9].

Clearly then, for the soft (bulk) sector in heavy ion collisions, geometrical issues dominate both the physics of interest and the system with which it is probed. No surprise, then, that since the relativistic heavy ion program began roughly two decades ago, femtoscopic studies have played a major role, and a "sub-community" has developed. It was not long before the erstwhile "nuclear" physicists contributed physical and technical insights to a type of measurement initially borrowed from their particle physics colleagues. At workshops like this, such dialogue continues unabated.

Several excellent reviews of femtoscopy in heavy ion physics have very recently appeared in the literature [1, 10, 11, 12]. Together with physics discussions, the reader may find in them precise definitions of the correlation function, "homogeneity lengths," "HBT radii," "out-side-long" coordinate system etc. Here, I assume familiarity with such concepts, and very briefly review the status of heavy ion femtoscopy at present. I emphasize the breadth of systematics which has been explored so far, what (we think) it has told us, and what continues to puzzle us. I then identify a few promising directions in which the field is moving, pointing for details on these to others' contributions to these proceedings.

\section{WHENCE}

Due to their copious production and ease of detection, most femtoscopic measurements have utilized correlations between charged pions. Further, many experiments have focused on central $(|\vec{b}|=0)$ collisions, since (1) azimuthal symmetry simplifies the femtoscopic formalism [12, 13]; and (2) maximal energy densities and spatial extents are generated. The extent of measured femtoscopic systematics 15-20 years ago is represented in Figure 1, showing that, in central collisions involving nuclei with mass number $A$, HBT radii scale approximately as $A^{1 / 3}[14,15]$. Apparently trivial, these data were at the same time comforting, confirming that pion correlations did indeed track with geometric scales. 


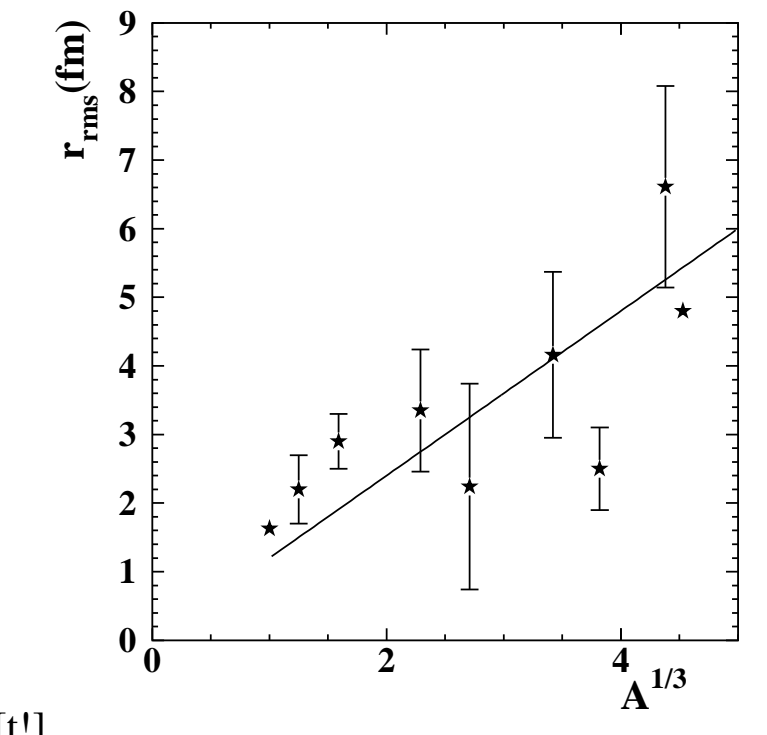

$[\mathrm{t} !]$

FIGURE 1. Pion HBT radius versus the mass FIGURE 2. World dataset of published HBT number of colliding nuclei, from Bevalac experi- radii from central $\mathrm{Au}+\mathrm{Au}(\mathrm{Pb}+\mathrm{Pb})$ collisions ments $\sim 20$ years ago. Compilation from [15].

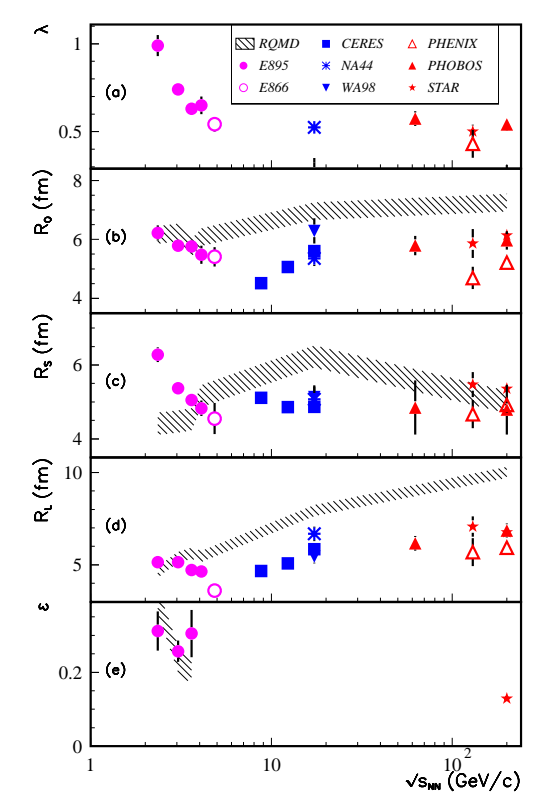

versus collision energy. Compilation from [12].

Since then, femtoscopic data and techniques have evolved tremendously, generating an equally tremendous range of systematic femtoscopic studies. The first femtoscopic measurement in truly relativistic heavy ion collisions was reported almost twenty years ago by the NA35 Collaboration at the CERN SPS [16]. Similar measurements have been performed at the SPS and the BNL AGS and RHIC accelerators over the collision energy range $\sqrt{s_{N N}} \approx 2.3-200 \mathrm{GeV}$. Thus, in each of the two complementary quantitiesenergy and time- we may consider two decades' worth of systematics [12].

The original hope was to find "anomalously" large spatial and/or temporal scales, as reflected in the HBT radii, indicating large entropy generation or a long-lived QGP state. This expectation was considered rather generic [17], and, guided by quantitative predictions from hydrodynamical models [3, 18], the most commonly-discussed systematic was the excitation function (i.e. energy dependence) of pion HBT radii. This is shown in Figure 2] where no striking features are observed in the HBT radii at any collision energy. As I discuss later, this observed contradiction of a seemingly-generic expectation may be considered the second "HBT puzzle."

Clearly, insight into geometrically-driven physics requires more detailed systematic studies than the simple excitation function. Indeed, this has always been a generic requirement for extracting physics from any observable in heavy ion physics, and has required development of heavy ion programs with simultaneous, complementary, largeacceptance experiments running at dedicated machines. Especially in the crucial soft sector, more is learned by varying independent variables than by long runs at the highest possible energy a given machine can deliver. 

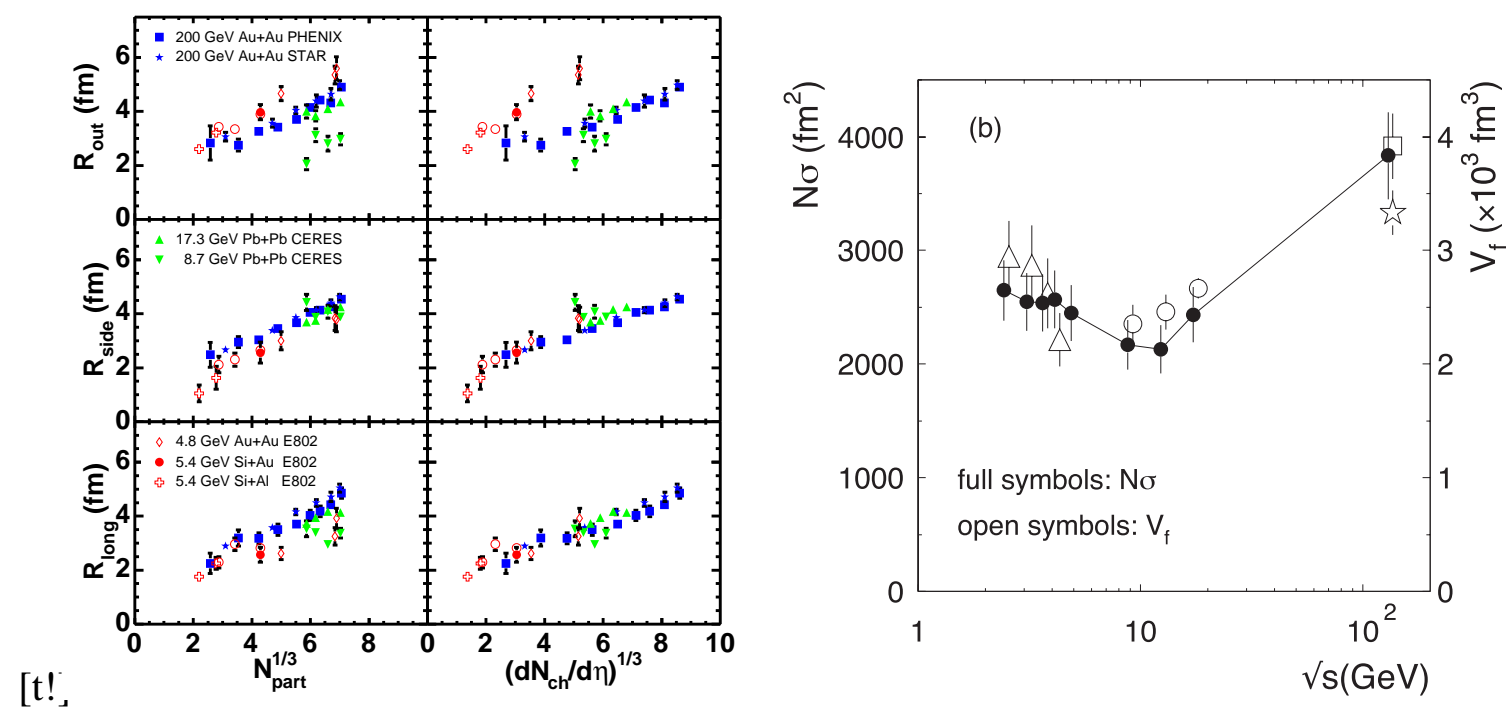

FIGURE 3. Pion HBT radii plotted versus the FIGURE 4. The "effective pion cross-section" number of participating nucleons (left panels), and $N_{\text {proton }} \cdot \sigma_{p \pi}+N_{\text {pion }} \cdot \sigma_{\pi \pi}$ and the "freezeout volversus the charged particle multiplicity (right pan- ume" $\sim R_{\text {long }} \cdot R_{\text {side }}^{2}$ are plotted as a function of els). Compilation from [12]. the collision energy, for central $\mathrm{Au}+\mathrm{Au}(\mathrm{Pb}+\mathrm{Pb})$ collisions. Figure and further details in [21].

Inspired by recent "schematic equations" [19], I denote the impressive multidimensional space explored by femtoscopic experiments as

$$
\text { Heavy Ion Femtoscopy }=R\left(\sqrt{s_{N N}} ; A, B,|\vec{b}|, \phi, y, m_{T}, m_{1}, m_{2}\right)
$$

Global dependences Especially in light of "puzzles," we need to perform a similar study as shown in Figure 11 checking that femtoscopic radii track with geometric collision scales to first order. We may vary the geometric scale of the reaction zone by varying the atomic numbers of the colliding nuclei, $A$ and $B$, and/or by selecting events of varying impact parameter, $|\vec{b}|$. Of course, fixing only one of these parameters will not define the collision scale; instead, a natural quantity would be the number of participating nucleons $N_{\text {part }}$ [20]. Pion HBT radii corresponding to different $A, B,|\vec{b}|$ and $\sqrt{s_{N N}}$ are collected in Figure 3 The left panels show that these femtoscopic lengths scale similarly to those shown in Figure 11 replacing $A$ by $N_{\text {part }}$. (Note that results for central collisions, $|\vec{b}| \approx 0$, are shown in Figure 1 so that $N_{\text {part }} \sim A$.) The HBT radius $R_{\text {out }}$, which mixes space and time non-trivially, may be expected to violate a pure geometrical scaling; this may explain the increased spread in the upper panels of Figure 3

To good approximation, at a given $\sqrt{s_{N N}}$, total multiplicity (a final-state quantity) is a function only of $N_{\text {part }}$ (an entrance-channel quantity), independent of $A, B$, or $|\vec{b}|$. The relationship does, however, depend on collision energy [22]. As seen in the right panels of Figure 3, the final-state multiplicity provides a more common scaling parameter than $N_{\text {part }}$; recent analyses [23, 24, 25] show that this scaling persists for different $m_{T}$ values 

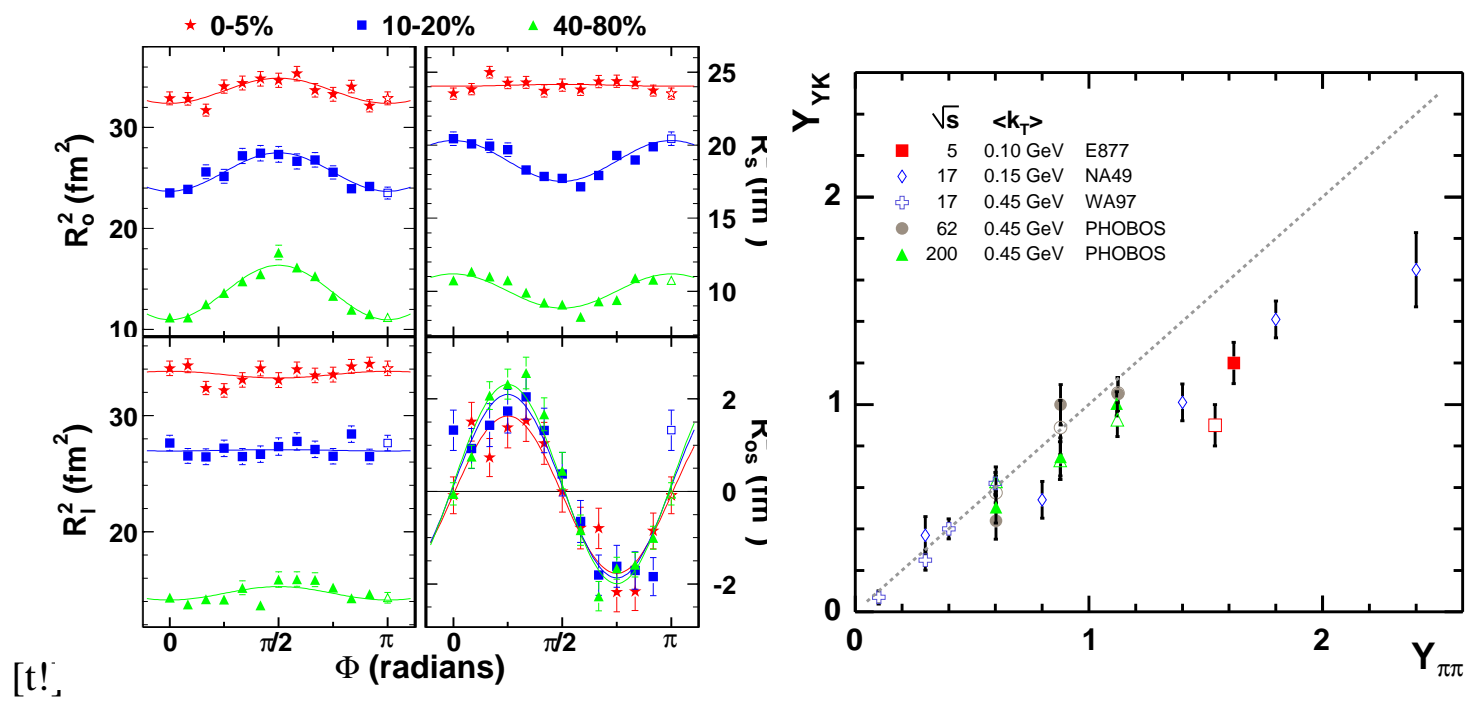

FIGURE 5. Pion HBT radii measured for FIGURE 6. The pion Yano-Koonin velocity $\mathrm{Au}+\mathrm{Au}$ collisions at $\sqrt{s_{N N}}$, plotted as a function (see text) versus pair rapidity for central $\mathrm{Au}+\mathrm{Au}$ of azimuthal emission angle relative to the reaction $(\mathrm{Pb}+\mathrm{Pb})$ collisions at various energies Compilation plane. From [27] from [12].

and for lighter colliding systems at RHIC.

Several observations may be made about this multiplicity scaling. Firstly, it appears that knowledge of $d N_{c h} / d \eta$ alone allows "prediction" of the HBT radii (at least $R_{\text {long }}$ and $R_{\text {side }}$ ). This suggests that the small increase of these radii with $\sqrt{s_{N N}}$ seen in Figure 2 is associated with increased particle production as the collision energy is raised. (Note that $N_{\text {part }}$ is approximately constant for the data in Figure 2]) Secondly, the finite offset $d$ in the approximately linear relationship $R_{\text {long }} \cdot R_{\text {side }}^{2}=c \cdot(d N / d \eta)+d$ means that freeze-out does not occur at fixed density [24].

Thirdly, the scaling shown in the figure breaks down dramatically for $\sqrt{s_{N N}} \lesssim 5 \mathrm{GeV}$, as is obvious from the non-monotonic behaviour seen in Figure 2 As the CERES Collaboration has pointed out [21], this is likely due to the dominance of baryons at lower $\sqrt{s_{N N}}$. Indeed, a quantitative connection between the number of protons and pions, and a product of HBT radii is possible, by assuming a universal ( $\sqrt{s_{N N}}$-independent) mean free path at freezeout $\lambda_{f}$. In Figure 4, the "freezeout volume" $\sim R_{\text {long }} \cdot R s i d e^{2}$ and the "effective pion cross-section" $N_{\text {proton }} \cdot \sigma_{p \pi}+N_{\text {pion }} \cdot \sigma_{\pi \pi}$ are seen to coincide by scaling the latter by $\lambda_{f}=1 \mathrm{fm}$, apparently contradicting the standard assumption that freeze-out occurs when the mean free path becomes much larger than the system size.

HBT radii and the "freeze-out volume" may be connected only the context of a model which includes dynamical effects like flow. The analysis of [21] ignores such effects; however, its bottom line remains approximately valid, as flow effects on HBT radii are expected to be small at low $p_{T}[26]$.

Kinematic dependences Insight on the dynamical evolution and geometric substructure of the emission region is gained by studying the dependence of femtoscopic lengths on the next three parameters in "Equation" 1.

In non-central collisions, the entrance-channel geometry is naturally anisotropic; the 


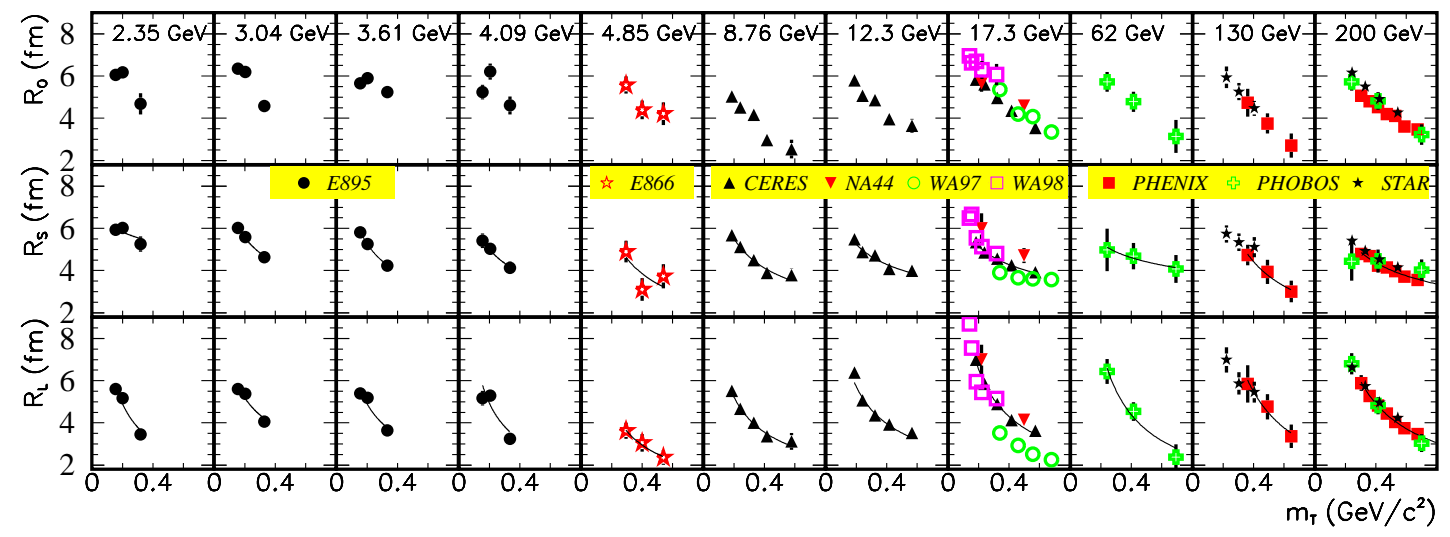

FIGURE 7. Pion HBT radii plotted versus the transverse mass $m_{T}$ for all published measurements of central $\mathrm{Au}+\mathrm{Au}(\mathrm{Pb}+\mathrm{Pb})$ collisions over two decades in $\sqrt{s_{N N}}$. Compilation from [12].

hot source geometry approximates the overlap between target and projectile, and is characterized by a "long axis" perpendicular to the impact parameter vector $\vec{b}$. At RHIC, the system expands more rapidly in-plane $(\| \vec{b})$ than out $(\perp \vec{b})[28]$. If it is, indeed a collective system with finite lifetime, then the overall shape should evolve. Pion HBT radii have been measured as a function of their azimuthal angle $\phi_{\text {pair }} \equiv \angle(\vec{K}, \vec{b})$ for $\mathrm{Au}+\mathrm{Au}$ collisions. The measurement at RHIC [27] is shown in Figure 5 There, it is clear that as $|\vec{b}| \rightarrow 0$, the freezeout source becomes larger and rounder. In fact, there is a nice "rule of two"- the source expands to twice its original size [23, 24], and its anisotropy $\varepsilon \equiv\left(\left\langle y^{2}\right\rangle-\left\langle x^{2}\right\rangle\right) /\left(\left\langle y^{2}\right\rangle+\left\langle x^{2}\right\rangle\right)$ decreases by the same factor [27]. The relatively small change in the source shape is at least semi-quantitatively [29] consistent with short timescale estimates [26] based on the longitudinal radius, and at variance with expectations from "realistic" simulations [30].

As will become increasingly clear, the only femtoscopic systematic which might display non-trivial $\sqrt{s_{N N}}$ dependence is, in fact, the dependence on $\phi_{p}$. This is clear from the bottom panel of Figure 2] in which the relative paucity of such measurements is also clear. It will be especially interesting to see whether the flow and/or timescales at the LHC are sufficiently large to produce in-plane freeze-out configurations [31].

Experiments at a wide range of collision energies have mapped out the rapidity dependence of pion HBT radii. Of particular interest here is the so-called Yano-Koonin rapidity $Y_{Y K}[32,33]$, which should approximate the rapidity of the fluid element which emits a pair of pions at some rapidity $Y_{\pi \pi}$. Figure 6 shows an approximately "universal" behaviour $Y_{\mathrm{YK}} \approx Y_{\pi \pi}$, independent of $\sqrt{s_{N N}}$. This is consistent with (but not proof of [12]) emission from a boost-invariant system [33].

The most extensively-studied kinematic systematic has been the $p_{T}$-dependence of pion HBT parameters. Figure 7 shows the world dataset of published measurements for central $\mathrm{Au}+\mathrm{Au}(\mathrm{Pb}+\mathrm{Pb})$ collisions. The falling dependence of femtoscopic scales on transverse velocity is generally believed to arise from collective transverse and 
$[\mathrm{t} !]$
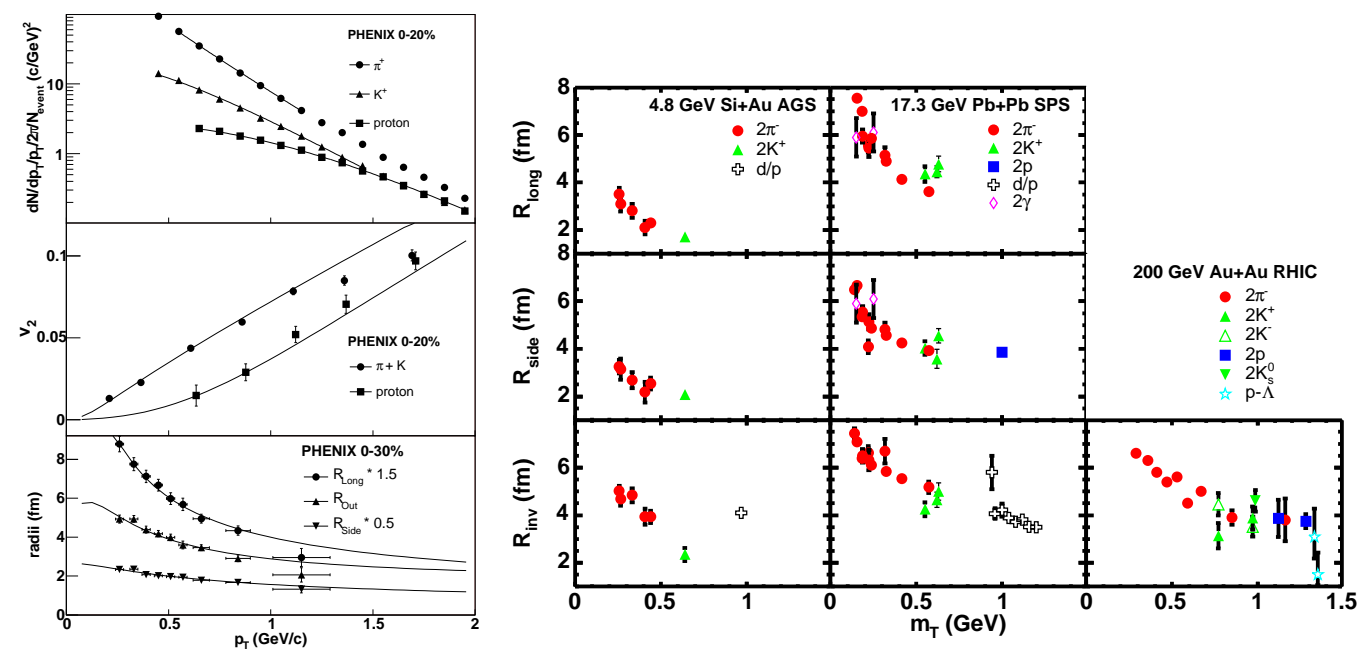

FIGURE 8. A blast-wave [26] fit repro- FIGURE 9. Femtoscopic radii for various similar-mass duces several observables at RHIC. See particle pairs, plotted as a function of $m_{T}$. Compilation text for details. From [34]. from [12].

longitudinal flow (e.g. [26]). As I mentioned earlier, strong collective flow would be an indication that a real bulk system has been formed.

The longitudinal radius scales approximately as $R_{l} \sim m_{T}^{-0.5}$, indicating strong longitudinal flow and again consistent with expectations for emission from a boost-invariant system [35, 26]. Decreasing transverse radii $R_{O}$ and $R_{S}$ may be due to collective transverse flow. The simplest flow-dominated models quantitatively interrelate these femtoscopic $m_{T}$ dependences with other observations. An example is shown in Figure 8 in which a very simple freeze-out scenario [26]- thermal motion superimposed on a collectively exploding source- can simultaneously describe a broad range of data measured at RHIC. The momentum-space distribution, quantified by the average number distribution (top panel of Figure 8) and the number variation as a function of azimuthal angle (middle panel) give an incomplete picture by themselves. Momentum-dependent femtoscopic radii (bottom panel) probe the dynamical $s u b$-structure of the collision, constraining models more stringently [12, 26, 36, 37].

Particle-species dependences Within the past several years, high-statistics datasets in experiments with good particle identification have allowed the mapping of femtoscopic systematics with the final variables in "Equation" 1- the mass (or species) of the correlated particles.

Signals of a system's collectivity at freeze-out should not be limited to the pions. In the simplest picture, corresponding to flow-dominated models (e.g. [26]) of Figure 8 , femtoscopic radii should approximately scale with $m_{T}$, independent of particle type. An impressive common scaling of radii from all measured particles is, indeed, observed at all energies explored, as seen in Figure 9. The common scaling is particularly striking when one considers the quite different measurement systematics involved in charged pion correlations and, say, $K_{s}^{0}$ correlations. Even generalized nucleon separation scales, probed by relative yields of deuterons and protons $(d / p$ in Figure 9), follow the systematic, with the exception of one outlier point at the lowest energies. 
TABLE 1. A very incomplete table of published or ongoing femtoscopic studies at RHIC for various particle combinations. "Traditional" identical-particle interferometry lies along the lowest diagonal line of cells.

\begin{tabular}{|c|c|c|c|c|c|c|c|c|c|c|}
\hline & $\pi^{+}$ & $\pi^{-}$ & $K^{+}$ & $K^{-}$ & $K_{s}^{0}$ & $p$ & $\bar{p}$ & $\Lambda$ & $\bar{\Lambda}$ & $\Xi$ \\
\hline $\bar{\Xi}$ & {$[38]$} & {$[38$} & & & & & & & & \\
\hline$\Xi$ & {$[38$} & {$[38]$} & & & & & & & & \\
\hline $\bar{\Lambda}$ & & & & & & {$[39]$} & [39] & & & \\
\hline $\bar{\Lambda}$ & & & & & & {$[39]$} & {$[\overline{39}]$} & & & \\
\hline $\bar{p}$ & $\overline{140} \overline{]}$ & {$[\overline{40}]$} & $\overline{140} \overline{1}$ & {$[\overline{40} \mid$} & & $|\overline{41}| \overline{\mid}$ & $\overline{41} \mid \overline{1}$ & & & \\
\hline$p$ & [40] & [40] & [40] & [40] & & [41, 42 & & & & \\
\hline$K_{s}^{0}$ & & & & & [43] & & & & & \\
\hline$\overline{K^{-}}$ & {$[\overline{44}]$} & [44] & & [42] & & & & & & \\
\hline$\overline{K^{+}}$ & [44] & {$[\overline{44}]$} & [42] & & & & & & & \\
\hline$\pi^{-}$ & $\lceil\overline{23}, \overline{41}] \overline{0}$ & {$[\overline{23}, \overline{45}, \overline{46}]$} & & & & & & & & \\
\hline $\bar{\pi}^{+}$ & {$[\overline{23}, \overline{45}, \overline{46}]$} & & & & & & & & & \\
\hline
\end{tabular}

Correlations between non-identical particles probe not only the sizes, but also the relative displacement of the particles' emission zones in space-time [47]. Any collective freeze-out scenario naturally implies a specific relationship between emission regions of the various particle types. In a flow-dominated picture, the emission zones for high$m_{T}$ particles are not only smaller than those for low- $m_{T}$ particles, but are also inevitably located further from the center of the collision region [26], as suggested by the schematic in Figure 10 As discussed in detail in the contribution of A. Kisiel [40], available measurements of these displacements at RHIC provide further support of the flowdominated freeze-out scenario.

Non-identical particle correlations are today a growth industry. Table 1 lists only a sampling of recently-published or ongoing analyses at RHIC energies. Similar studies have been performed at lower energies [48, 12]. The diagonal axis corresponds to identical-particle correlations, the "traditional" focus of HBT interferometry.

\section{WHITHER}

Excluding aficionados attending workshops such as this one, in the minds of most heavy ion (or high energy) physicists, the term "femtoscopy" (or, more likely "HBT") brings to mind only the single, rather uninspiring systematic plotted in Figure 2 indeed, some may be tempted toward the dismissive view that "the measured radius is always $5 \mathrm{fm}$."

As we have just discussed, this is grossly unfair: the systematics are tremendously richer, with femtoscopic length scales varying with almost every parameter in "Equation" 1. Furthermore, these strong systematic trends are found consistently by experiments separated by decades and using quite different measurement and correction techniques; indeed, especially at RHIC, the data are almost embarrassingly consistent (c.f. Figure (7). Yet further, it appears that these systematics may be well understood in the commonly-accepted framework of system evolution due to strong flow quantitatively consistent with momentum-space observables [26]. Clearly, there is much more to fem- 


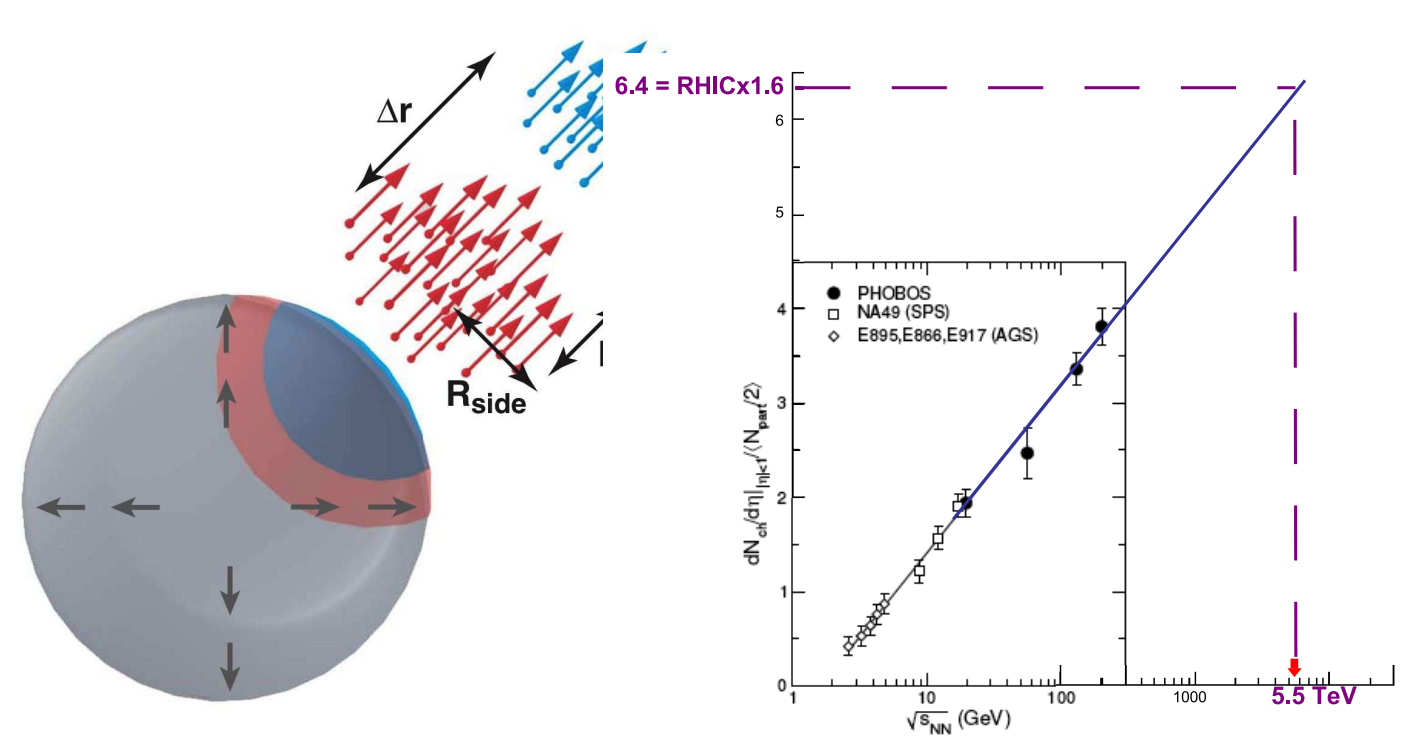

FIGURE 10. Freezeout regions for particles of FIGURE 11. Multiplicity density per participant different species (or different transverse masses) pair, measured for $\mathrm{Au}+\mathrm{Au}(\mathrm{Pb}+\mathrm{Pb})$ collisions at emitted from a common source. Two-particle AGS, SPS, and RHIC are shown in the lower panel, correlations measure the (momentum-dependent) taken from [22], and naively extrapolated by the size, shape, and orientation of the emission re- author to LHC energy.

gions, as well as the average displacement $(\Delta r)$ in the outward direction. From [12].

toscopy than its most notorious Figure.

On the other hand, the trends shown in Figures 3 , 6, 7 and 9 suggest that the notorious Figure 2 quite correctly summarizes the situation after all. At any $\sqrt{s_{N N}}$, the systematics of "Equation" 1 are quite rich and may well be reconciled with a reasonable physical picture. However, in more ways than expressed by Figure 2, those systematics are essentially independent of $\sqrt{s_{N N}}$ ! Without resorting to agreement or disagreement with particular models, this second ${ }^{2}$ femtoscopic puzzle is startling, suggesting that the space-time consequences of the physical processes are the same at RHIC as they are near the pion production threshold. Often, "universal" behaviour is a key to deeper physical insight. Heavy ion femtoscopy, however, might display a bit too much universality.

\section{Whither... or wither?}

2 In this experimental overview, I have not discussed what has come to be known as "the" HBT puzzle [49] which, simply put, is that otherwise-successful and apparently reasonable models like hydrodynamics do not reproduce femtoscopic measurements [12]. To all but the novice heavy ion physicist, however, the initial failure of dynamical models to reproduce diverse observations is hardly puzzling. The experience at lower energies is that such initial failure is more the rule than the exception. In light of other, more generic puzzles, I call this problem only the "first HBT puzzle" [37]. 
Given the prominence of nontrivial geometry to the physics of heavy ion collisions in general, and the rather generic [17] expectation of significant changes in spacetime evolution with $\sqrt{s_{N N}}$, understanding this universality remains urgent. What future efforts might shed some light?

Today, one almost reflexively points to the impending heavy ion program at the LHC for new observations generating fresh insights. While anything might happen in an unexplored energy domain, we may venture a prediction. Instead of a crystal ball, however, we use a mirror to gaze over our shoulder at twenty years of systematics in heavy ions. The $\sqrt{s_{N N}}$-dependence of the global multiplicity (per participant pair) has been significantly extended at RHIC and summarized by the PHOBOS collaboration [22]. Boldly and probably naively extending this systematic leads to the expectation that multiplicities at the LHC will be $\sim 60 \%$ higher than they are at RHIC, as shown in Figure 11

As discussed in the previous Section, femtoscopic length scales- for any $m_{T}, y, N_{\text {part }}$, or particle species- depend primarily on event multiplicity. Taken together, Figures 11 and 3 suggest that radii ${ }^{3}$ in central collisions at RHIC will simply be $\sim 17 \%$ higher than they are at RHIC $\left((1.6)^{1 / 3}=1.17.\right)$.

Notably, evidence is mounting that perhaps all soft-sector observables are determined primarily by total multipilcity, independent of $\sqrt{s_{N N}}$. Properly-scaled elliptic flow [50] and even strangeness enhancement [51] appear to show universal multiplicity scalings. Whether this is a trivial implication of entropy-driven phasespace dominance in observables sensitive to bulk medium is unclear. However, nontrivial new phases of matter should have signatures in the long-distance (soft momentum) sector; dependence only on multiplicity and not reaction energy would be intriguing.

So, perhaps the choice of collider facility (LHC versus RHIC) is unimportant, and heavy ion femtoscopists should focus on filling in the holes of Table 11. Most evidence thus far indicates that flow-dominated freezeout scenarios (e.g. [26]) fitted to identical $\pi$ correlations essentially "predict" femtoscopic data using other particle combinations. The data is yet scant, however, so ongoing studies [40] to further explore this Table are quite important. There are even preliminary reports [38], with exotic particle combinations, of inconsistencies with these freezeout models. If confirmed, strong theoretical focus should come to bear on this result. If, on the other hand, varying the particle combination repeatedly yields results "predicted" by blast-wave models, continually filling in cells of Table 1 risks becoming a stamp-collecting exercise.

The most important recent experimental developments in heavy ion femtoscopy were presented at these workshops. In addition to those discussed above, I briefly mention here some ongoing studies which I find most promising.

Even if all of the particle combinations in Table 1 follow simple blast-wave calculations, and so reveal no new femtoscopic information, this can actually be turned to good use. In particular, one may turn around the traditional approach in which one uses the known the two-particle final state interaction (FSI) to extract geometric information, to extract the FSI itself [48, 52]. Finalized results from STAR on $p-\Lambda$ correlations [39]

\footnotetext{
${ }^{3}$ Precise expectations $R_{\text {out }}$ or $\phi$-dependent radii at the LHC are, admittedly, less certain, as the former does not scale exactly with multiplicity (cf Figure 3), and the multiplicity-dependence of the latter has not been extensively mapped (cf Figures 2 and 5 .
} 
have extracted previously inaccessible phase shift information for low-energy baryonantibaryon scattering. While not QGP-related physics, such studies can make a unique contribution to low-energy QCD and hadronic physics.

Ideally suited workshops like these are studies which directly compare for the first time, at a fixed energy and using identical detector and analysis techniques, correlation data from the heaviest ion collisions to that from $p+p$ collisions [24]. As has been observed previously in high energy experiments, femtoscopic radii from identical pion correlations measured in $\mathrm{p}+\mathrm{p}$ collisions decrease with increasing $p_{T}$, qualitatively similar to the dependence shown in Figure 7 The preliminary STAR data shows, however, that in all three HBT radii, the $p_{T}$ dependence is quantitatively identical in $\mathrm{p}+\mathrm{p}$ and $\mathrm{A}+\mathrm{A}$ collisions! Since the heavy ion and high energy communities have traditionally used very different physics mechanisms to explain this dependence, this observation potentially throws the explanations of both communities into doubt. If this result is confirmed, it ranks as the "third HBT puzzle" [37].

Unexplained long-range structure in the correlation functions for the lowestmultiplicity collisions, however, presently cloud the interpretation of the HBT radii for $p+p$ collisions [24]. Partly in an effort to understand this, a new representation of the data in terms of spherical harmonic amplitudes in $\vec{q}$-space was developed as an experimental diagnostic tool [37]. In fact, a similar harmonic decomposition method was earlier already developed by Danielewicz and collaborators [53, 54], not as a diagnostic, but as a direct link to the detailed geometry (beyond simply length scales) of the emitting source. This representation has a natural connection to source imaging [55], and, indeed, first applications to PHENIX data have been reported [56].

Harmonic decompositions as an improved representation of the correlation function and source imaging as an improved, generalized fit to the data are, in a sense, merely technical improvements, but they are quite significant ones. Just as femtoscopic studies have explored the systematic landscape of Equation 11 so should they probe the "microscape" of fine details of the measured data.

The femtoscopy of heavy ion collisions can be an addicting endevour. Systematics make sufficient sense that we are convinced that we are probe geometry at the femtometer scale. Spacetime geometry at that scale is sufficiently important to the physics that the measurements must be done well. Such measurements are sufficiently challenging that it is enjoyable to do them well and to develop improved techniques. However, for now, the overall results are sufficiently puzzling that there is plenty more to do.

\section{ACKNOWLEDGMENTS}

I express my sincere gratitude to the organizers of both ISMD and WPCF. Both actually succeeded in what is often just a stated but unattained goal of such workshops: to achieve meaningful intellectual cross-pollenation between nuclear and particle physicists. I express special congratulations to the initiators of WPCF, which itself sprang out of the earlier Warsaw meetings, for establishing a timely and most promising workshop series. 


\section{REFERENCES}

1. R. Lednicky (2005), nucl-th/0510020

2. M. Gyulassy, and L. McLerran, Nucl. Phys. A750, 30-63 (2005), nucl-th/ 0405013

3. D. H. Rischke, and M. Gyulassy, Nucl. Phys. A608, 479-512 (1996), nucl-th/9606039

4. P. Huovinen, P. F. Kolb, U. W. Heinz, P. V. Ruuskanen, and S. A. Voloshin, Phys. Lett. B503, 58-64 (2001), hep-ph/0101136

5. J.-Y. Ollitrault, Nucl. Phys. A638, 195-206 (1998), nucl-ex/9802005

6. J. Adams, et al., Phys. Rev. Lett. 93, 252301 (2004), nucl-ex/ 0407007

7. B. Cole, plenary presentation at Quark Matter 2005, Budapest (2005).

8. R. J. Fries, B. Muller, C. Nonaka, and S. A. Bass, Phys. Rev. Lett. 90, 202303 (2003), nucl-th/0301087

9. D. Molnar, Acta Phys. Hung. A22, 271-279 (2005), nucl-th/0406066

10. T. Csorgo (2005), nucl-th/0505019

11. S. S. Padula, Braz. J. Phys. 35, 70-99 (2005), nucl-th/0412103

12. M. A. Lisa, S. Pratt, R. Soltz, and U. Wiedemann, Ann. Rev. Nucl. Part. Sci. 55, 311 (2005), nucl-ex/0505014

13. U. W. Heinz, A. Hummel, M. A. Lisa, and U. A. Wiedemann, Phys. Rev. C66, 044903 (2002), nucl-th/0207003

14. J. Bartke, Phys. Lett. B174, 32-35 (1986).

15. G. Alexander, Rept. Prog. Phys. 66, 481-522 (2003), hep-ph/0302130

16. T. J. Humanic, et al., Z. Phys. C38, 79-84 (1988).

17. J. W. Harris, and B. Muller, Ann. Rev. Nucl. Part. Sci. 46, 71-107 (1996), hep-ph/9602235

18. S. A. Bass, et al., Nucl. Phys. A661, 205-260 (1999), nucl-th/9907090

19. M. Gyulassy, J. Phys. G30, S911-S918 (2004).

20. A. Bialas, M. Bleszynski, and W. Czyz, Nucl. Phys. B111, 461 (1976).

21. D. Adamova, et al., Phys. Rev. Lett. 90, 022301 (2003), nucl-ex/0207008

22. B. B. Back, et al., Nucl. Phys. A757, 28-101 (2005), nucl-ex/0410022

23. J. Adams, et al., Phys. Rev. C71, 044906 (2005), nucl-ex/ 0411036

24. Z. Chajecki, contribution to these proceedings (2005).

25. Z. Chajecki (2005), nucl-ex/0510014

26. F. Retiere, and M. A. Lisa, Phys. Rev. C70, 044907 (2004), nucl-th/ 0312024

27. J. Adams, et al., Phys. Rev. Lett. 93, 012301 (2004), nucl-ex/0312009

28. J. Adams, et al., Phys. Rev. Lett. 92, 062301 (2004), nucl-ex/0310029

29. M. A. Lisa, Acta Phys. Polon. B35, 37-46 (2004), nucl-ex/0312012

30. D. Teaney, J. Lauret, and E. V. Shuryak (2001), nucl-th/0110037

31. U. W. Heinz, and P. F. Kolb, Phys. Lett. B542, 216-222 (2002), hep-ph/0206278

32. F. B. Yano, and S. E. Koonin, Phys. Lett. B78, 556-559 (1978).

33. Y. F. Wu, U. W. Heinz, B. Tomasik, and U. A. Wiedemann, Eur. Phys. J. C1, 599-617 (1998), nucl-th/9607044

34. F. Retiere, J. Phys. G30, S827-S834 (2004), nucl-ex/ 0405024

35. S. V. Akkelin, and Y. M. Sinyukov, Phys. Lett. B356, 525-530 (1995).

36. B. Tomasik (2005), nucl-th/0509100

37. Z. Chajecki, T. D. Gutierrez, M. A. Lisa, and M. Lopez-Noriega (2005), nucl-ex/0505009

38. P. Chaloupka, contribution to these proceedings (2005).

39. J. Adams (2005), nucl-ex/0511003

40. A. Kisiel, contribution to these proceedings (2005).

41. H. Gos, contribution to these proceedings (2005).

42. M. Heffner, J. Phys. G30, S1043-S1047 (2004).

43. S. Bekele, J. Phys. G30, S229-S234 (2004).

44. J. Adams, et al., Phys. Rev. Lett. 91, 262302 (2003), nucl-ex/0307025

45. S. S. Adler, et al., Phys. Rev. Lett. 93, 152302 (2004), nucl-ex/ 0401003

46. B. B. Back, et al. (2004), nucl-ex/0409001

47. R. Lednicky (2001), nucl-th/0112011

48. R. Lednicky, contribution to these proceedings (2005).

49. U. W. Heinz, and P. F. Kolb (2002), hep-ph/0204061. 
50. G. Roland, et al. (2005), nucl-ex/0510042

51. H. Caines, poster presentation at Quark Matter 2005, Budapest (2005).

52. M. Bystersky, and F. Retiere, contribution to these proceedings (2005).

53. P. Danielewicz, and S. Pratt (2005), nucl-th/0501003

54. P. Danielewicz, contribution to these proceedings (2005).

55. D. Brown, contribution to these proceedings (2005).

56. P. Chung, contribution to these proceedings (2005). 